\title{
Long lasting structural changes in primary motor cortex after motor skill learning: a behavioural and stereological study
}

\author{
PAOLA MORALES ${ }^{1}$
}

${ }^{1}$ Programme of Molecular \& Clinical Pharmacology, ICBM, Medical Faculty, University of Chile

\begin{abstract}
Many motor skills, once acquired, are stored over a long time period, probably sustained by permanent neuronal changes. Thus, in this paper we have investigated with quantitative stereology the generation and persistence of neuronal density changes in primary motor cortex (MI) following motor skill learning (skilled reaching task). Rats were trained a lateralised reaching task during an "early" (22-31 days old) or "late" (362371 days old) postnatal period. The trained and corresponding control rats were sacrificed at day 372 , immediately after the behavioural testing. The "early" trained group preserved the learned skilled reaching task when tested at day 372, without requiring any additional training. The "late" trained group showed a similar capacity to that of the "early" trained group for learning the skilled reaching task. All trained animals ("early" and "late" trained groups) showed a significant inter hemispheric decrease of neuronal density in the corresponding motor forelimb representation area of MI (cortical layers II-III).

It is concluded that learning a skilled reaching task implies long lasting structural changes in restricted cortical regions of the motor cortex. The generation and persistence of these changes probably reflect a plastic reorganization for storing and retrieving motor skills. The plastic changes were also observed in the older rats, suggesting that motor cortex maintains its plastic capacity throughout the lifespan.
\end{abstract}

Key terms: Motor cortex, plasticity, persistence of structural changes, aging, motor skill learning (skilled reaching task), stereology, rat.

\section{INTRODUCTION}

From the behavioural point of view, it is well known that many motor skills, once acquired, are preserved for a long time. There is experimental evidence in mammals indicating that after motor training, functional (Karni et al. 1998; Kleim et al. 1998; Pascual-Leone et al. 1994; Plautz et al. 2000; Rioult-Pedotti et al. 2007, Derksen et al. 2007) and structural (Anderson et al. 2002; Díaz et al. 1994; Greenough et al. 1985; Kleim et al. 1996; Morales et al. 1999; Withers and Greenough 1989) changes occur in the primary motor cortex (MI). The persistence of some of these changes in the absence of continued training has been a focus for a great number of investigations (see Monfils 2005). Indeed, it has been shown that motor learning induces an enlarged local blood oxygenation area in the human MI, persisting after several months without requiring further training (Karni et al. 1995). Similarly, rats trained a complex motor task show an increase in the number of synapses per Purkinje cell, which persisted after 28 days (Kleim et al. 1997). In a previous study, rats trained for a skilled reaching task during 10 days, starting at the age of 22 or 62 days, showed a significant reduction of neuronal density on the contralateral forelimb motor representation area $(15 \%$ and $13 \%$ respectively; Morales et al. 1999). That reduction persisted after a month without any training, suggesting a plastic mechanism by which procedural memory was preserved.

Correspondence (CA): Paola Morales Retamales, PhD., MSci, Programme of Molecular \& Clinical Pharmacology, ICBM, Medical Faculty, University of Chile, P.O.Box 70.000, Santiago 7, Chile. Email: pmorales@med.uchile.cl, Phone number: (56)-2-678 6788, Fax (56)-2-7372783 
The purpose of the present study was to evaluate: (i) the long-term persistence of a morphological change (neuronal density) induced by a skilled reaching task trained at early-stages (22 days old), and (ii) the ability of aged rats (360 days old) to learn a skilled reaching task, developing a structural plasticity similar to that shown by juvenile (22 days old) rats.

The present study has been carried out to honour the memory of my teacher Prof. Dr. Teresa Pinto-Hamuy, a pioneering and inspiring scholar for a generation of scientists interested in behavioural neuroscience.

\section{MATERIALS AND METHODS}

\section{Subjects}

Sixteen male gray rats $(\mathrm{AxC})$ from a local colony were randomly allocated to either (i) an "early" trained group (trained for 10 days, during the 22 to 31 days of age period; $n=6$ ), or (ii) a "late" trained group (trained for 10 days, during the 362 to 371 days of age period, $n=6$ ). Untrained control subjects were matched by age to each trained group ( $n=6$, for each condition). Rats were individually housed, kept in a 12 hr day/night cycle with ad libitum access to water. During the training the rats were food restricted and maintained at 85-90\% of their ad libitum feeding weight, in order to increase the level of motivation required to solve a task (Saavedra et al. 1999).

All procedures were approved by a Local National Committee for Ethics for Experiments with Laboratory Animals at the Medical Faculty, University of Chile (TP-H, DID S9608/2: DID SOO-01/2) endorsing the Principles of laboratory animal care (NIH; No 86-23; revised 1985).

\section{Training box:}

Two home-made wooden testing boxes were used, one for early post-weaned $\left(17 \times 20 \times 20 \mathrm{~cm}^{3}\right)$, and another for aged $\left(27 \times 27 \times 30 \mathrm{~cm}^{3}\right)$ rats. The front wall of each chamber had a glass panel and a 1.3 or a 1.7 $\mathrm{cm}$ wide slot, respectively.

\section{Skilled reaching task training}

Only animals showing a lateral preference (right or left paw) for solving a reaching task were selected as experimental subjects (more than $90 \%$ of preference, as in previous reports; Díaz et al. 1994; Morales et al. 1999). The selected animals were trained for 10 days, four sessions per day. Each session implied 25 trials, making a total of 1000 trials, as described before (Díaz et al. 1994; Morales et al. 1999). A trial consisted of an attempt by the rat with its preferred paw to reach and grasp a sweet cereal grain held at the tip of a hatpin. The sweet cereal grain was far enough from the box to require the animal to perform a full extension of its forelimb. Control rats were exposed to an identical training box, for the same number of sessions, but food was available on the floor of the box. At the end of the training the rats were individually housed, kept in a $12 \mathrm{hr}$ day/night cycle with ad libitum access to water and food, and their behaviour was periodically monitored until the day 372. At day 372, control and "early" trained rats were tested for one session consisting of 25 trials, to verify the manual preference and/or persistence of the learned skilled-reaching task, respectively.

\section{Histological procedure}

After the last testing session (day 372), the rats were deep anaesthetised with chloral hydrate $(0.5 \mathrm{ml}, 7 \%$ solution; i.p. $)$ and perfused intracardially with a $0.9 \%$ saline solution followed by a $4 \%$ formaldehyde/ saline solution. The brains were removed immediately, post-fixed and cryoprotected for $48 \mathrm{hr}$ in a $30 \%$ sucrose solution. Frozen serial coronal sections (30 $\mu \mathrm{m}$ thick) were obtained using a sliding microtome and stained with cresyl violet (Merck, Darmstadt, Germany), dehydrated and mounted with Entellan (Merck).

\section{Regional sampling}

The sampling procedure is illustrated in Fig. 1. In A, a schematic representation of the rat brain is shown, indicating the area from where slices were taken (MI is 
represented by the parallelepiped inserted through coronal sections from Bregma 2.7 to Bregma -0.4). In B, the sampling area is depicted (inserted square) at $20 \mathrm{x}$ magnification. In $\mathrm{C}$, the optical disector is shown at $100 x$ magnification.

\section{Sterology}

The stereological evaluation of neuronal density was conducted in the bright field of a light microscope (Nikon 83888) as previously described (Morales et al., 1999), using the optical disector technique (Sterio 1984; West and Gundersen 1990). The sampling from the hemispheres was matched, taking one out of three serial sections, counting the neuronal nuclei with an ocular micrometer grid under $100 x$ oil immersion objective. The criteria for neuronal recognition were according to Ling et al. (1973) and Braendgaard et al. (1990). Neuronal nuclei were counted as they came into the focus of the microscope, while scanning through a section. Neurons were identified by a pale nucleus with a prominent nucleolus. Smaller chromatin condensed nuclei, characteristic of glial cells, were not counted. For each section, six unbiased counting frames were sampled in a randomized fashion inside an area of $0.11 \mathrm{~mm}^{2}$. This area was situated $2.5 \mathrm{~mm}$ from the cerebral midline in order to avoid sampling from the cingulate cortex and lateral somatosensory neurons (see Fig $1 \mathrm{~A}$, B). The area of the disector (adis) was set at

(A)

(B)

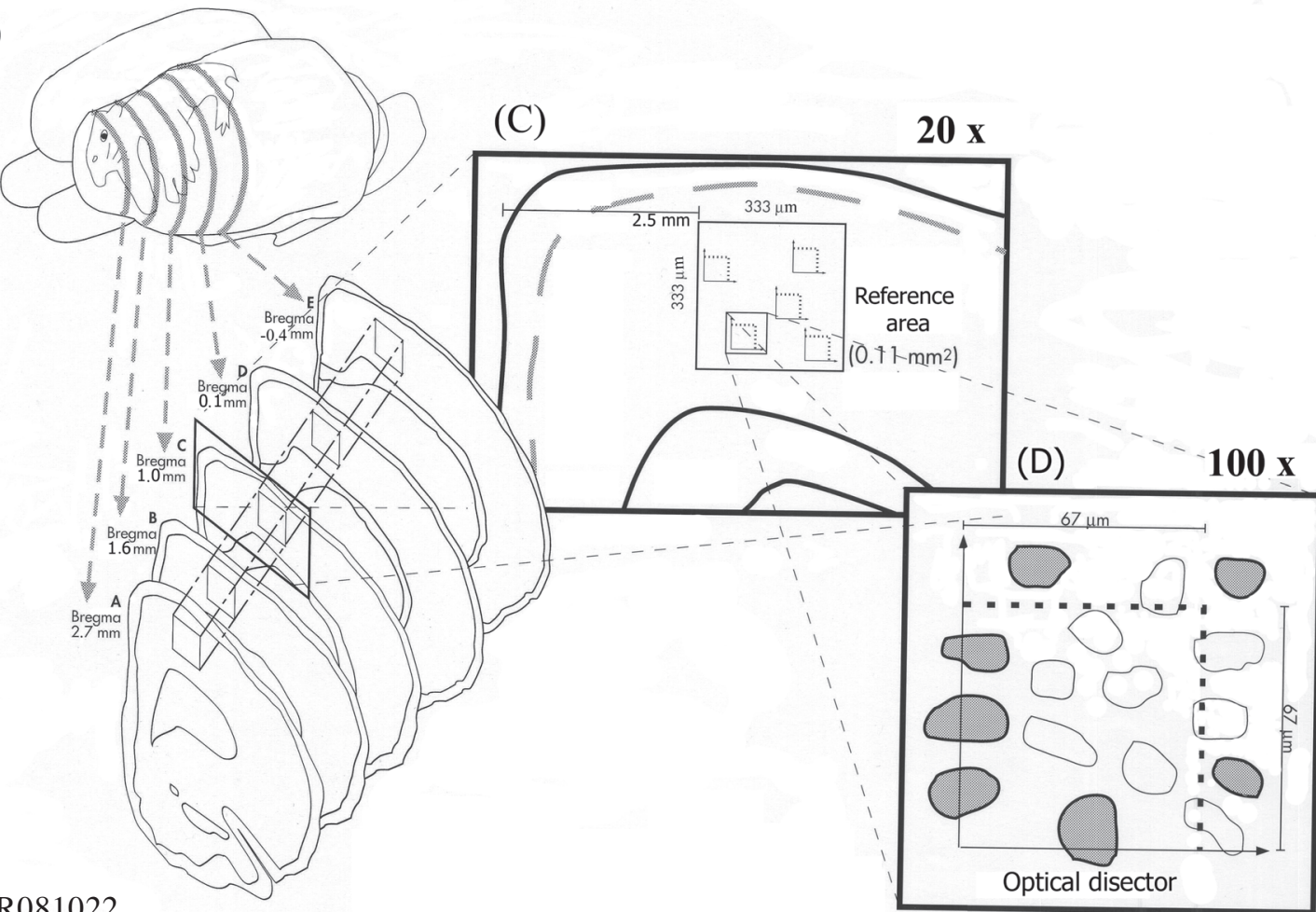

Figure 1: Sampling procedure. (A) Shows a schematic diagram of the MI, including the rat equivalent of a homunculus, ratunculus. Samples were taken from Bregma 2.7 to $-0.4 \mathrm{~mm}, 2.5 \mathrm{~mm}$ from the midline in order to avoid sampling from the cingulate cortex. The parallelepiped indicates the tri-dimensional extension of the MI where the counting was performed. In (B), the sampled area in layers II-III is shown at 20x. In (C), the application of the optical disector for nuclei selection is shown. At 100x, cell nuclei were identified, selected and counted as shown in the Materials and Methods section. Six randomly selected optical dissectors were used for each reference area, counting only nuclei (open, in the drawing) inside the optical dissector. A total of 187 individual scores per hemisphere for each subject were analyzed. 
$4.6 * 10^{3} \mu \mathrm{m}^{2}$. The disector height (h) was set at $8 \mu \mathrm{m}$ and nuclei within the first $3 \mu \mathrm{m}$ of the section were not counted. As well, we discarded all the nuclei that intersected the left and the bottom sides of the frame (see Fig. 1C).

The neuronal density was estimated as:

$\mathrm{Nv}=Q^{-} / h^{*} a_{d i s}$

Where $\mathrm{Q}^{-}$is the total average of nuclei counted, $\mathrm{h}$, the disector height, $\mathrm{a}_{\mathrm{dis}}$, the area of disector.

Neuronal counting was performed by two observers, who were not aware of the experimental designations of the brains.

\section{Statistical analysis}

Values are expressed as the means \pm standard error of the means (S.E.M.) throughout the study. In a within-subject hemisphere comparison, the "trained hemisphere" was defined as the one contralateral to the used forelimb, while the "untrained hemisphere" corresponded to the ipsilateral. The one-tailed-paired Student's t-test was used for the within-subjecthemisphere-comparison, while the onetailed-unpaired-Student's t-test was used for the between group comparison (GraphPad Prism; version 4.00; 2003; S. Diego, CA, USA). The level $\mathrm{p}<0.05$ was used as a limit for statistical significance.

\section{RESULTS}

\section{Behavioural analysis: skilled reaching task}

Trained and control groups showed a natural bias for the use of the right forelimb in approximately $70 \%$ of the cases $(n=24)$ when exposed to an experimental condition requesting a skilled reaching task, consisting of reaching and grasping a sweet cereal grain offered and handled by the experimenter. An "early' trained group $(n=6)$ was first challenged with learning the task for 10 days, starting at day 22. At the end of the training, the rats were housed (three per cages) in a $12 \mathrm{hr}$ day/night cycle with ad libitum access to water and food, and their behaviour was periodically monitored until day 372. During that period, the rats displayed bilateral forelimb performance for feeding, grooming, playing and any other basic behaviors, according to their species strategies. When the "early" trained rats were tested for the skilled reaching task at day 372, they immediately made an attempt to reach and grasp a sweet cereal with their preferred paws. The rats retrieved the skill learned earlier without a new session of training. The "late" trained group (trained for 10 days, starting at day $362 ; n=6$ ) learned and performed the task in a similar manner to that of the "early" trained group in terms of speed, handedness and accuracy.

\section{Morphological analysis: neuronal density}

As shown in table 1, the stereological analysis of neuronal density evaluated at day 372 did not show any interhemispheric differences in untrained control animals, regardless of handedness or age when the animals were exposed to the training box (i.e. "early"- or "late"-period, $n=6$, for each condition). However, in "early" and "late" trained animals, there was a significant decrease of neuronal density when comparing the trained (contralateral to the used forelimb) versus the untrained hemisphere (ipsilateral to the used forelimb). The decrease in neuronal density was observed at the level of Bregma 1.5-0.1, in both "early" $(18.21 \pm 1.77 \%, p<0.005 ; \mathrm{n}=6)$ and "late" $(16.39 \pm 1.38 \%, p<0.005 ; \mathrm{n}=6)$ trained animals, both analysed at day 372 . Neuronal density observed in the untrained hemisphere of "early" and "late" trained animals was not different to that observed in the controls. However, a decrease in neuronal density was also observed when comparing the trained versus the untrained control hemispheres ("early", $17.1 \pm 1.34 \%$, $p<0.005 ; \mathrm{n}=6$ and "late" $12.3 \pm 1.45 \%, p<$ $0.005 ; n=6)$, (see Table 1$)$.

\section{DISCUSSION}

A motor skill is generally agreed as a learned skill that involves voluntary 
movement to perform a task. Rats are already able to learn complex motor skills at early developmental stages if properly challenged. Indeed, soon after opening their eyes, rats are able to identify an appetitive stimulus, targeting it as a possible food source and co-ordinately reaching and grasping it by using a preferred paw (Díaz et al, 1994). A successfully selected skilled reaching task can be incorporated into the behavioural repertoire of an animal, to be available for future challenges, depending upon the extent and the efficacy of the training period. That incorporation into the behavioural repertoire implies plastic changes that may occur in a particular brain region. A behavioural repertoire also assumes innate preferences for performing an act. Thus, it was found here that 22-dayold rats showed a natural bias for the right forelimb for reaching a difficult to access source of appetitive food. The stored skilled reaching task can be retrieved when the animal is exposed to a similar condition, as in this case at day 372, when the animal was tested for the skilled-reaching task.

Learning and retrieval of a motor skill task implies plastic changes in the motor cortex (Anderson et al. 2002; Díaz et al.
1994; Greenough et al. 1985; Kleim et al. 1996; Withers and Greenough 1989, Kleim et al. 2004). In agreement, in a previous study, rats trained for a skilled reaching task at early stages (22 or 62 days) showed a decrease in neuronal density in cortical layers II-III of the MI (Díaz et al. 1994, Morales et al. 1999). In the present study, a similar decrease was found in neuronal density in the MI of the trained hemisphere, compared to the untrained hemisphere of "early" trained rats examined at day 372. This finding indicates that learning and retrieval of a motor skill task imprints long lasting structural changes in MI. That plastic change was also observed when the skilled reaching task was trained and evaluated at a senior stage, suggesting that motor cortex preserves a plastic capacity throughout the lifespan. The structural changes were restricted to a region which, according to electrophysiological representation maps (Sanes and Donoghue 2000; Sanes et al. 1990; Donoghue and Wise 1982; Neafsey et al. 1986) corresponds to the forelimb cortex. No differences in neuronal density were found in the rostral or caudal adjacent areas. Control rats did not show any cortical

TABLE I

Average of neuronal density $\left(\times 10^{4}\right.$ neurons $\left./ \mathrm{mm}^{3} \pm \mathrm{SEM}\right)$ found in the rostral, medial and caudal region ( $\mathrm{mm}$ with respect to Bregma, $\mathrm{B}$ ) of the layer II-III of MI.

\begin{tabular}{|c|c|c|c|c|c|c|}
\hline & \multicolumn{3}{|c|}{ Hemisphere ipsilateral to the used forelimb } & \multicolumn{3}{|c|}{ Hemisphere contralateral to the used forelimb } \\
\hline & B 2.4 to 1.2 & B 1.5 to 0.1 & B 0 to -0.4 & B 2.4 to 1.6 & B 1.5 to 0.1 & B 0 to -0.4 \\
\hline \multicolumn{7}{|l|}{ Control } \\
\hline "early" & $23.12 \pm 0.40$ & $25.56 \pm 0.38$ & $27.89 \pm 0.33$ & $22.83 \pm 0.37$ & $25.14 \pm 0.48$ & $27.29 \pm 0.51$ \\
\hline "late" & $23.22 \pm 0.43$ & $25.87 \pm 0.64$ & $27.91 \pm 0.49$ & $23.61 \pm 0.38$ & $26.00 \pm 0.73$ & $28.21 \pm 0.60$ \\
\hline \multicolumn{7}{|l|}{ Trained } \\
\hline "early" & $21.27 \pm 0.68$ & $25.62 \pm 0.95$ & $27.06 \pm 0.37$ & $20.44 \pm 0.58$ & $\underline{20.95} \pm \underline{0.91^{\mathrm{a}, \mathrm{b}}}$ & $26.49 \pm 0.63$ \\
\hline "late" & $23.52 \pm 0.54$ & $27.18 \pm 0.61$ & $28.83 \pm 0.53$ & $23.30 \pm 0.41$ & $\underline{22.81} \pm \underline{0.82} \underline{\mathrm{a}, \mathrm{b}}$ & $28.69 \pm 0.69$ \\
\hline
\end{tabular}

There was a significant decrease of neuronal density in the hemisphere contralateral to the used forelimb at the level of Bregma 1.5-0.1, compared to the ipsilateral hemisphere, in both "early" and "late" trained groups The difference was also significant when the trained hemisphere was compared to that observed in the corresponding "early" and "late" untrained control animals. Data are means \pm S.E.M. a=p $<0.005$ versus the untrained hemisphere; $b=p<0.005$ versus corresponding hemispheres from controls. 
asymmetry, regardless of handedness and the age when the rats were tested. Therefore, the changes shown here were learned specific. There are studies showing that neither repetition of unskilled movements (Kleim et al. 1998; Plautz et al 2000), training strength (Remple et al. 2001), exercise training (Kleim et al. 2002), nor lack of a sufficiently extensive motor skill training (Kleim el al. 2004) are sufficient to induce permanent changes in motor cortex, as observed in the present report.

The decrease in neuronal density found here might be a consequence of neuropile growth following increased connectivity and/or strengthening of existing ones (Rioult-Pedotti et al. 2000,1998; Morales et al. 1999; Keller et al. 1992; Withers and Greenough 1989; Greenough et al. 1985). This interpretation is supported by Golgi studies, demonstrating an enlargement of apical dendrites in layers II-III (Withers and Greenough 1989) and in V (Greenough et al. 1985) of the somatosensory-motor cortex after a similar motor task. In a study quantifying MAP-2 (microtubule associated protein-2)-positive dendrites, an increase of dendrite branching was found in layers IIIII and V of MI after a motor skill-learning task (Bury and Jones 2002). Moreover, an increased expression of MAP-2, synaptophysin (a synapses formation marker) (Derksen et al. 2007) and brainderived neurotrophic factor (BDNF) were also observed after complex motor learning (Klintsova et al. 2004), suggesting changes in neuronal morphology and connectivity. Synaptogenesis in layers II-III of MI has also been found after motor skill learning (Kleim et al. 2002; Kleim et al. 2004; Kleim et al. 1996) and after long-term potentiation (LTP) of MI pathways (Keller et al. 1992). The strengthening of horizontal connections from somatosensory cortex (SI) to layers II-III of MI via an LTP-like mechanism has been demonstrated for motor skilled training (Pavlides et al. 1993; Martin and Morris 2001; Monfils and Testey 2004; Rioult-Pedotti et al. 2007; 2000; 1998). These long-lasting changes suggest synaptogenesis (Kleim et al. 1998), dendritic spine expansion (Harms et al. 2008) and/or reorganization of the representation maps in MI (Kleim et al. 2004; 1998; Sanes and Donoghue 2000). There is an inverse relationship between cortical thickness and cellular density following environmental stimulation (Beaulieu and Colonnier 1989). In agreement, there is a significant increase of cortical thickness and a decrease of cellular density following a reaching motor task at an early post-weaned period (Díaz et al. 1994), as well as in adulthood (Anderson et al. 2002). Hence, it can be suggested that a decrease in neuronal density reflects an increase of dendritic arborisation and synaptogenesis, implying changes in cortical volume. To our knowledge, no apoptosis has been observed following motor training, but we cannot rule out that other factors, such as angiogenesis (Kleim et al. 2002) and/or gliosis are increased (Li et al. 2005; Sirevaag and Greenough 1991), perhaps contributing to the decreased neuronal density reported here.

Many studies have documented that aging in humans (Harrington and Haaland 1992; McNay and Willingham 1998; Seidler 2006) and rats (Markus and Petit, 1987) implies a reduced ability for skill learning. In many cases, even when training is for a long period, the performance does not reach the skill level shown by young adult subjects. However, in the present study, "late" trained (one year old) rats were able to learn and perform a skilled reaching task, as well as young rats, suggesting that the motor cortex maintains a plastic capacity in adulthood. It has been demonstrated recently that in humans seniors can learn "to learn" new motor skills, as well as young people (Seidler 2007). However, the mechanisms involved in the acquisition of a new motor skill at senior stages are still unknown.

In conclusion, learning a skilled reaching task implies long-lasting morphological changes within a restricted cortical region of the motor cortex (MI). Those changes were also observed in older animals, suggesting that the acquisition of new motor skills at that period of life is possible. The capacity to acquire new motor skills could be essential for adaptive motor function throughout the lifespan. 


\section{ACKNOWLEDGEMENTS}

The authors thank Professor Dr. Mario Herrera-Marschitz for his review and inspired comments while we prepared the manuscript. Mr. Aníbal Martinez is acknowledged for his excellent technical assistance. This work was supported by Grants DID SOO-01/2 (TP-H), Fondecyt 11070192 (PMR).

\section{REFERENCES}

ANDERSON BJ, ECKBURG PB, RELUCIO KI (2002) Alterations in the thickness of motor cortical subregions after motor-skill learning and exercise. Learn Mem 9: 1-9.

BEAULIEU C, COLONNIER M (1989) Effects of the richness of the environment on six different cortical areas of the cat cerebral cortex. Brain research 495: 382-386.

BRAENDGAARD H, EVANS SM, HOWARD CV, GUNDERSEN HJ (1990) The total number of neurons in the human neocortex unbiasedly estimated using optical disectors. J Microsc 157: 285-304.

BURY SD, JONES TA (2002) Unilateral sensorimotor cortex lesions in adult rats facilitate motor skill learning with the "unaffected" forelimb and traininginduced dendritic structural plasticity in the motor cortex. J Neurosci 22: 8597-8606.

DERKSEN MJ, WARD NL, HARTLE KD, IVANCO TL (2007) MAP2 and synaptophysin protein expression following motor learning suggests dynamic regulation and distinct alterations coinciding with synaptogenesis. Neurobiol Learn Mem 87: 404-415.

DÍAZ E, PINTO-HAMUY T, FERNANDEZ V (1994) Interhemispheric structural asymmetry induced by a lateralized reaching task in the rat motor cortex. Eur $\mathrm{J}$ Neurosci 6: 1235-1238.

DONOGHUE JP, WISE SP (1982) The motor cortex of the rat: cytoarchitecture and microstimulation mapping. $\mathrm{J}$ Comp Neurol 212: 76-88.

GREENOUGH WT, LARSON JR, WITHERS GS (1985) Effects of unilateral and bilateral training in a reaching task on dendritic branching of neurons in the rat motorsensory forelimb cortex. Behav Neural Biol 44: 301314.

HARMS KJ, RIOULT-PEDOTTI MS, CARTER DR, DUNAEVSKY A (2008) Transient Spine Expansion and Learning-Induced Plasticity in Layer 1 Primary Motor Cortex. J Neurosci 28: 5686-5690.

HARRINGTON DL, HAALAND KY (1992) Skill learning in the elderly: diminished implicit and explicit memory for a motor sequence. Psychol Aging 7: 425-434.

KARNI A, MEYER G, JEZZARD P, ADAMS MM, TURNER R, UNGERLEIDER LG (1995) Functional MRI evidence for adult motor cortex plasticity during motor skill learning. Nature 377: 155-158.

KARNI A, MEYER G, REY-HIPOLITO C, JEZZARD P, ADAMS MM, TURNER R, UNGERLEIDER LG (1998) The acquisition of skilled motor performance: fast and slow experience-driven changes in primary motor cortex. PNAS 95: 861-868.

KELLER A, ARISSIAN K, ASANUMA H (1992) Synaptic proliferation in the motor cortex of adult cats after long-term thalamic stimulation. J Neurophysiol 68: 295-308.

KLINTSOVA AY, DICKSON E, YOSHIDA R, GREENOUGH WT (2004) Altered expression of BDNF and its high-affinity receptor TrkB in response to complex motor learning and moderate exercise. Brain Res 1028: 92-104.

KLEIM JA, HOGG TM, VANDENBERG PM, COOPER NR, BRUNEAU R, REMPLE M (2004) Cortical synaptogenesis and motor map reorganization occur during late, but not early, phase of motor skill learning. J Neurosci 24: 628-633.

KLEIM JA, BARBAY S, COOPER NR, HOGG TM, REIDEL CN, REMPLE MS, NUDO RJ (2002) Motor learning-dependent synaptogenesis is localized to functionally reorganized motor cortex. Neurobiol Learn Mem 77: 63-77.

KLEIM JA, BARBAY S, NUDO RJ (1998) Functional reorganization of the rat motor cortex following motor skill learning. J Neurophysiol 80: 3321-3325

KLEIM JA, VIJ K, BALLARD DH, GREENOUGH WT (1997) Learning-dependent synaptic modifications in the cerebellar cortex of the adult rat persists for at least four weeks. J Neurosci 17: 717-721.

KLEIM JA, LUSSNIG E, SCHWARZ ER, COMERY TA, GREENOUGH WT (1996) Synaptogenesis and Fos expression in the motor cortex of the adult rat after motor skill learning. J Neurosci 16: 4529-4535.

LI J, DING YH, RAFOLS JA, LAI Q, MCALLISTER JP, 2ND, DING Y (2005) Increased astrocyte proliferation in rats after running exercise. Neurosci Lett 386: 160164.

LING EA, PATERSON JA, PRIVAT A, MORI S, LEBLOND CP (1973) Investigation of glial cells in semithin sections. I. Identification of glial cells in the brain of young rats. J Comp Neurol 149: 43-71.

MARKUS EJ, PETIT TL (1987) Neocortical synaptogenesis, aging, and behavior: lifespan development in the motor-sensory system of the rat. Exp Neurol 96: 262-278.

MARTIN SJ, MORRIS RG (2001) Cortical plasticity: It's all the range! Curr Biol 11: R57-59.

MCNAY EC, WILLINGHAM DB (1998). Deficit in learning of a motor skill requiring strategy, but not of perceptuomotor recalibration, with aging. Learn Mem 4: 411-420.

MONFILS MH, PLAUTZ EJ, KLEIM JA (2005) In search of the motor engram: motor map plasticity as a mechanism for encoding motor experience. Neuroscientist 11: 471-483.

MONFILS MH, TESKEY GC (2004) Skilled-learninginduced potentiation in rat sensorimotor cortex: a transient form of behavioural long-term potentiation Neuroscience 125: 329-336.

MORALES P, PINTO-HAMUY T, FERNÁNDEZ V, DÍAZ E (1999) Persistent neuronal density changes related to the establishment of a motor memory. Behav Brain Res 99: 115-121.

NEAFSEY EJ, BOLD EL, HAAS G, HURLEY-GIUS KM, QUIRK G, SIEVERT CF, TERREBERRY RR (1986) The organization of the rat motor cortex: a microstimulation mapping study. Brain Res 396: 77-96.

PASCUAL-LEONE A, GRAFMAN J, HALLETT M. 1994. Modulation of cortical motor output maps during development of implicit and explicit knowledge. Science 263: 1287-1289.

PAVLIDES C, MIYASHITA E, ASANUMA H. 1993. Projection from the sensory to the motor cortex is important in learning motor skills in the monkey. $\mathrm{J}$ Neurophysiol 70: 733-741 
PLAUTZ EJ, MILLIKEN GW, NUDO RJ. 2000. Effects of repetitive motor training on movement representations in adult squirrel monkeys: role of use versus learning. Neurobiol Learn Mem 74: 27-55.

REMPLE MS, BRUNEAU RM, VANDENBERG PM, GOERTZEN C, KLEIM JA (2001) Sensitivity of cortical movement representations to motor experience: evidence that skill learning but not strength training induces cortical reorganization. Behav Brain Res 123: 133-141.

RIOULT-PEDOTTI MS, DONOGHUE JP, DUNAEVSKY A (2007) Plasticity of the synaptic modification range. J Neurophysiol 98: 3688-3695.

RIOULT-PEDOTTI MS, FRIEDMAN D, DONOGHUE JP (2000) Learning-induced LTP in neocortex. Science 290: 533-536.

RIOULT-PEDOTTI MS, FRIEDMAN D, HESS G, DONOGHUE JP (1998) Strengthening of horizontal cortical connections following skill learning. Nat Neurosci 1: 230-234.

SANES JN, DONOGHUE JP (2000) Plasticity and primary motor cortex. Ann Rev Neurosci 23: 393-415.

SAAVEDRA M, PINTO-HAMUY T, MARCHANT F, URZUA C (1999) Motivación: su efecto sobre la estrategia de solución del laberinto octagonal. Revista de Psicología de la Universidad de Chile 8: 143-159.
SANES JN, SUNER S, DONOGHUE JP (1990) Dynamic organization of primary motor cortex output to target muscles in adult rats. I. Long-term patterns of reorganization following motor or mixed peripheral nerve lesions. Exp Brain Res 79: 479-491.

SEIDLER RD (2007) Older adults can learn to learn new motor skills. Behav Brain Res 183: 118-122.

SEIDLER RD (2006) Differential effects of age on sequence learning and sensorimotor adaptation. Brain Res Bull 70: 337-346.

SIREVAAG AM, GREENOUGH WT (1991) Plasticity of GFAP-immunoreactive astrocyte size and number in visual cortex of rats reared in complex environments. Brain Res 540: 273-278.

STERIO DC (1984) The unbiased estimation of number and sizes of arbitrary particles using the disector. J Microsc 134: 127-136.

WEST MJ, GUNDERSEN HJ (1990) Unbiased stereological estimation of the number of neurons in the human hippocampus. J Comp Neurol 296: 1-22.

WITHERS GS, GREENOUGH WT (1989) Reach training selectively alters dendritic branching in subpopulations of layer II-III pyramids in rat motor-somatosensory forelimb cortex. Neuropsychologia 27: 61-69. 